\title{
ARTICLE Alcohol intake enhances glutamatergic transmission from D2 receptor-expressing afferents onto D1 receptor-expressing medium spiny neurons in the dorsomedial striatum
}

\author{
Jiayi Lu ${ }^{1}$, Yifeng Cheng ${ }^{1}$, Xuehua Wang ${ }^{1}$, Kayla Woodson ${ }^{1}$, Craig Kemper ${ }^{1}$, Emily Disney ${ }^{1}$ and Jun Wang (iD ${ }^{1}$
}

\begin{abstract}
Dopaminergic modulation of corticostriatal transmission is critically involved in reward-driven behaviors. This modulation is mainly mediated by dopamine D1 receptors (D1Rs) and D2Rs, which are highly expressed in medium spiny neurons (MSNs) of the dorsomedial striatum (DMS), a brain region essential for goal-directed behaviors and addiction. D1Rs and D2Rs are also present at presynaptic cortical terminals within the DMS. However, it is not known how addictive substances alter the glutamatergic strength of striatal synapses expressing presynaptic dopamine receptors. Using cell type-specific Cre mice in combination with optogenetic techniques, we measured glutamatergic transmission at D1R- or D2R-expressing afferents to DMS MSNs. We found larger excitatory postsynaptic currents at the synapses between the extra-striatal D2R-expressing afferents and D1R-expressing MSNs (D2 $\rightarrow$ D1), as compared with those observed at the other tested synapses (D1 $\rightarrow \mathrm{D} 1, \mathrm{D} 1 \rightarrow \mathrm{D} 2$, and D2 $\rightarrow \mathrm{D} 2)$. Additionally, excessive alcohol consumption induced a long-lasting potentiation of glutamatergic transmission at the corticostriatal D2 $\rightarrow$ D1 synapse. Furthermore, we demonstrated that activation of postsynaptic, but not presynaptic, D2Rs inhibited corticostriatal transmission in an endocannabinoid-dependent manner. Taken together, these data provide detailed information on the mechanisms underlying dopamine receptor-mediated modulation of brain reward circuitry.
\end{abstract}

Neuropsychopharmacology (2019) 44:1123-1131; https://doi.org/10.1038/s41386-019-0332-9

\section{INTRODUCTION}

Dopamine signaling in the corticostriatal circuit is centrally involved in reward-driven behaviors [1]. By acting on different glutamatergic terminals, striatal dopamine regulates specific excitatory inputs from the cortex and thalamus onto medium spiny neurons (MSNs), contributing to the selection of appropriate behaviors [1]. Dopaminergic modulation is mainly mediated by dopamine D1 receptors (D1Rs) and D2Rs [2, 3], which are highly expressed in the dorsomedial striatum (DMS), a brain region essential for goal-directed behaviors and addiction [4, 5]. DMS MSNs express D1Rs or D2Rs [6] and anatomical evidence indicates that these receptors are present at presynaptic cortical terminals in the striatum $[7,8]$. These presynaptic receptors facilitate dopamine-mediated regulation of excitatory signaling to specific striatal pathways [1]. Altered dopaminergic transmission is a common mechanism underlying drug and alcohol abuse [9, 10]. However, it is not known how excessive alcohol consumption affects glutamatergic synapses expressing particular pre- and postsynaptic dopamine receptors.

The medial prefrontal cortex (mPFC) afferent input to the DMS has been extensively implicated in drug and alcohol addiction [11-13]. Excessive alcohol exposure has been reported to enhance glutamatergic responses at MPFC inputs to the DMS [14], and this enhancement was recently shown to contribute to a long-lasting enhancement of alcohol-seeking and alcohol-taking behaviors [15]. The neuronal subtypes within the MPFC can also be distinguished based on their expression of D1Rs and D2Rs [2].
However, it is not known how excessive alcohol consumption distinctly alters D1R- or D2R-expressing mPFC afferents onto striatal MSNs.

In this study, we measured the effects of excessive alcohol intake on glutamatergic transmission at DMS synapses expressing different pre- and postsynaptic dopamine receptors. We discovered that synapses containing presynaptic D2Rs and postsynaptic D1Rs (D2 $\rightarrow$ D1) exhibited stronger glutamatergic connectivity than the other tested synapses $(\mathrm{D} 1 \rightarrow \mathrm{D} 1, \mathrm{D} 1 \rightarrow \mathrm{D} 2$, and $\mathrm{D} 2 \rightarrow \mathrm{D} 2)$. Excessive alcohol intake induced a long-lasting potentiation of glutamatergic transmission at the corticostriatal D2 $\rightarrow$ D1 synapse. In addition, we demonstrated that D2R-mediated inhibition of glutamatergic transmission in the DMS is mediated by distinct preand postsynaptic mechanisms. These findings contribute to the elucidation of the detailed mechanisms underlying the dopaminergic modulation of different brain reward circuitry. Our results also identified alcohol-evoked circuit-specific plasticity in the DMS, which may contribute to excessive alcohol consumption.

\section{MATERIALS AND METHODS}

Details are provided in the Supplemental Materials and Methods.

\section{Reagents}

The viral vector was purchased from the University of North Carolina Vector Core. Reagents were obtained from Sigma, R\&D Systems, and Abcam.

${ }^{1}$ Department of Neuroscience and Experimental Therapeutics, College of Medicine, Texas A\&M University Health Science Center, Bryan, TX, USA

Correspondence: Jun Wang (jwang@medicine.tamhsc.edu)

Received: 1 March 2018 Revised: 12 January 2019 Accepted: 30 January 2019

Published online: 7 February 2019 


\section{Animals}

The D1-Cre and D2-Cre mice were obtained from Mutant Mouse Regional Resource Centers. Ai32, Ai14, and Snap25 mice were purchased from the Jackson Laboratory. All animal care and experimental procedures were approved by the Texas A\&M University Institutional Animal Care and Use Committee.

\section{Stereotaxic infusion}

AAV-Flex-Chronos-GFP or AAV-CAG-Flex-GFP was bilaterally infused into the $\mathrm{mPFC}(\mathrm{AP},+1.94 \mathrm{~mm} ; \mathrm{ML}, \pm 0.25 \mathrm{~mm} ; \mathrm{DV}$, $-2.5 \mathrm{~mm}$ from the Bregma) of D1-/D2-Cre;Ai14 mice. AAVretroCAG-Tdtomato was bilaterally infused into the DMS (AP1, +1.18 $\mathrm{mm}$; ML1, $\pm 1.3 \mathrm{~mm}$; DV1, $-2.9 \mathrm{~mm} ; \mathrm{AP2},+0.38 \mathrm{~mm} ; \mathrm{ML} 2, \pm 1.67$ $\mathrm{mm}$; DV2, $-2.9 \mathrm{~mm}$ from the Bregma) of D1-/D2-Cre;Snap25 mice.

\section{Intermittent-access to $20 \%$ alcohol two-bottle-choice drinking procedure}

Mice were trained to consume $20 \%$ alcohol for 8 weeks using the intermittent-access two-bottle-choice drinking procedure as previously described $[2,5,16,17]$.

\section{Electrophysiology}

Slice preparation and electrophysiological recordings have been described previously $[2,5,15,16,18]$. Slices were maintained in external solution containing (in $\mathrm{mM}$ ): $125 \mathrm{NaCl}, 4.5 \mathrm{KCl}, 2.5 \mathrm{CaCl}_{2}$, $1.3 \mathrm{MgCl}_{2}, 1.25 \mathrm{NaH}_{2} \mathrm{PO}_{4}, 25 \mathrm{NaHCO}_{3}, 15$ glucose, and 15 sucrose. The electrodes contained (in mM): $119 \mathrm{CsMeSO}_{4}, 8 \mathrm{TEA} . \mathrm{Cl}, 15$ HEPES, 0.6 EGTA, $0.3 \mathrm{Na}_{3} \mathrm{GTP}, 4 \mathrm{MgATP}, 5 \mathrm{QX}-314$, and 7 phosphocreatine (for voltage-clamp recording) or 123 potassium gluconate, 10 HEPES, 0.2 EGTA, $8 \mathrm{NaCl}, 2 \mathrm{MgATP}, 0.3 \mathrm{NaGTP}$ (for

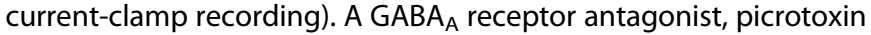
$(100 \mu \mathrm{M})$, was included in the external solution for all recordings, in order to block GABAergic transmission.

\section{Histology and cell counting}

Fluorescent images were acquired using a confocal microscope (FluoView 1200, Olympus, Tokyo, Japan) and analyzed using IMARIS 8.3.1 (Bitplane, Zürich, Switzerland), as previously reported [2].

\section{Statistical analysis}

Data were analyzed by two-tailed $t$ test (unpaired or paired), oneway analysis of variance (ANOVA), or two-way ANOVA with repeated measures (two-way RM ANOVA), followed by the Student-Newman-Keuls (SNK) post hoc test.

\section{RESULTS}

DMS glutamatergic synapses containing different pre- and postsynaptic dopamine receptors exhibit distinct connectivity To achieve selective activation of D1R- or D2R-expressing neurons, we crossed D1-Cre or D2-Cre mice, in which Cre expression was driven by the D1R or D2R promotor, with a channelrhodopsin-2 (ChR2)-EYFP Cre reporter line, Ai32 [19, 20]. We recently verified that Cre expression in these mouse lines reliably represented D1Rand D2R-expressing neurons outside the striatum [2]. In D1-Cre; $\mathrm{Ai} 32$ and D2-Cre;Ai32 mice, we observed intense expression of EYFP in the dorsal striatum and at the projection targets of D1-MSNs and D2-MSNs (Fig. 1a, b), confirming ChR2 expression in these neurons. We utilized a biophysical approach to identify MSN subtypes. In D1-Cre;Ai32 mice, D1-MSNs expressed ChR2 and responded distinctly to 2-ms and 500-ms light stimulations (Figure S1Ai, S1B) [21]. Conversely, D2-MSNs in these animals did not express ChR2, but received glutamatergic afferents expressing D1Rs and ChR2; in these cells, 500-ms light stimulation did not significantly alter the width of the response to 2-ms light stimulation (Figure S1Aii, S1B). The same approach was used to identify D1-MSNs and D2-MSNs in D2-Cre;Ai32 mice (Figure S1C).
Next, we measured the light-evoked response in the presence of an AMPAR antagonist, DNQX $(10 \mu \mathrm{M})$, and found that the responses at $\mathrm{D} 1 \rightarrow \mathrm{D} 2$ and $\mathrm{D} 2 \rightarrow \mathrm{D} 1$ synapses were completely blocked (Figure S1D, S1E). In contrast, the light-evoked responses of $\mathrm{D} 1 \rightarrow \mathrm{D} 1$ or $\mathrm{D} 2 \rightarrow \mathrm{D} 2$ were partially suppressed due to a large postsynaptic ChR2-mediated depolarization (Figure S1F). This ChR2mediated direct depolarization may cause a space-clamp error, which affects the accuracy of measurements of D1 $\rightarrow D 1$ and D2 $\rightarrow$ D2 connectivity. To overcome this space-clamp issue, we measured the strontium $\left(\mathrm{Sr}^{2+}\right)$-induced asynchronous excitatory postsynaptic currents (aEPSCs) evoked by light stimulation [22, 23]. We found that the aEPSC frequency was significantly higher at the $D 2 \rightarrow D 1$ synapse than at the other three synapse types (Fig. 1c, d; $\left.F_{(3,49)}=32.88, p<0.001\right)$, and that the aEPSC amplitude was identical in these four types of synapse (Fig. $1 c, e^{2} F_{(3,49)}=0.14, p>0.05$ ).

The higher aEPSC frequency indicated that the D2 $\rightarrow$ D1 synapse may show the strongest connectivity. To confirm this, we compared the light-evoked AMPAR-EPSCs at D1 $\rightarrow$ D2 and $\mathrm{D} 2 \rightarrow \mathrm{D} 1$ synapses; these can be measured directly, without interference from postsynaptic ChR2-mediated depolarization (Fig. 1f). We found significantly larger EPSC amplitudes at $\mathrm{D} 2 \rightarrow \mathrm{D} 1$ synapses than at $\mathrm{D} 1 \rightarrow \mathrm{D} 2$ synapses (Fig. 1g; $t_{(16)}=$ $-2.543, p<0.05)$. Lastly, we confirmed that D1-Cre and D2-Cre mice crossed with reporter lines expressed the same proportions of D1R- and D2R-expressing neurons (Figure S2).

Together, these results suggest that DMS glutamatergic synapses containing different pre- and postsynaptic dopamine receptors exhibit distinct connectivity, and that the D2 $\rightarrow$ D1 connectivity is stronger than that of the other tested connections.

DMS-projecting extra-striatal neurons preferentially express D2Rs We next explored the potential mechanism underlying the relatively greater strength of the DMS D2 $\rightarrow$ D1 synapse. Previous anatomical studies indicated that cortical fibers in the dorsal striatum contain abundant D2Rs, and fewer D1Rs [7, 8]. It was therefore possible that there were more D2R-expressing fibers, as compared with those expressing the D1R. To test this, we employed D1-Cre;Snap25 and D2-Cre;Snap25 mice where D1Rand D2R-expressing neurons expressed GFP, respectively (Fig. 2a). A retrograde virus encoding tdTomato (AAVretro-CAG-tdTomato) was infused into the DMS in order to label DMS-projecting neurons (Fig. 2a). Co-expression of tdTomato and GFP was visualized as a yellow signal (Fig. 2a-d).

We observed that more of the MPFC projections to the DMS expressed the D2R, as compared with the D1R (Fig. 2b). We counted yellow neurons in other extra-striatal areas of both hemispheres throughout the entire brain. To compensate for infusion and expression variations, we normalized the number of yellow neurons to the number of red neurons in the MPFC. This analysis of brain-wide inputs to the DMS found that significantly more DMS-projecting neurons expressed the D2R, as compared with the total number of neurons that expressed the D1R (Fig. 2e; $\left.t_{(4)}=-3.03, p<0.05\right)$. Additionally, many individual brain sections contained significantly more D2R-expressing neurons than D1Rexpressing cells (Fig. 2f; $F_{(1,130)}=8.92, p<0.05$ ). Interestingly, we discovered that the posterior brain regions tended to have more D2R- than D1R-expressing DMS-projecting neurons (Fig. 2g; $t_{(4)}=$ $-4.03, p<0.05)$, while the anterior regions showed no significant differences between the D1R-expressing and D2R-expressing cells (Fig. $2 g ; t_{(4)}=-2.04, p>0.05$ ). These data suggest that extra-striatal D2R-expressing inputs to the DMS are more prevalent than D1Rexpressing inputs, and that presynaptic D2Rs may therefore play a stronger regulatory role than D1Rs.

Suppression of glutamatergic transmission at distinct DMS synapses via pre- and postsynaptic D2Rs

D2R-mediated inhibition plays a critical role in modulating corticostriatal glutamatergic transmission. Although the 

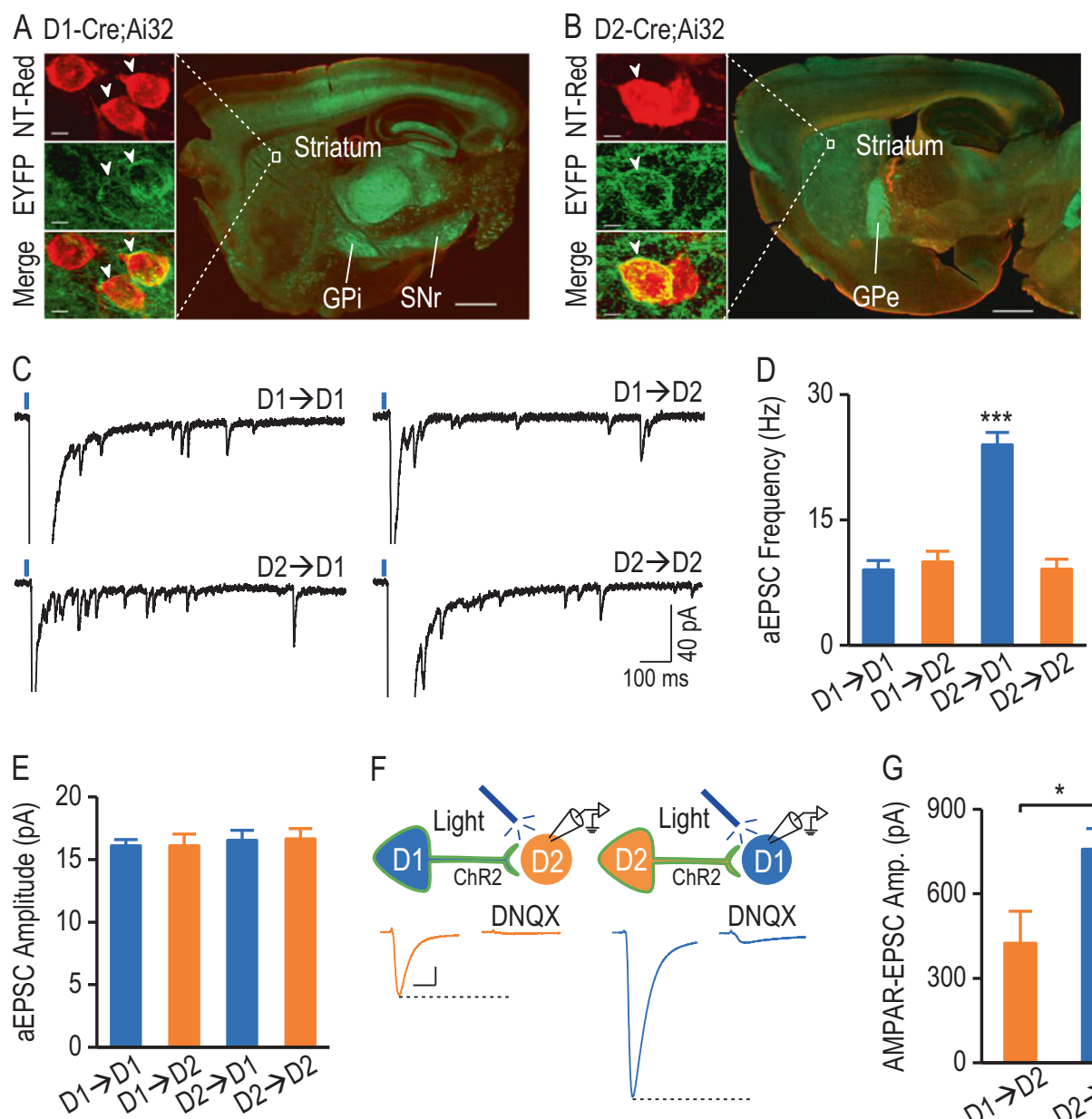

F

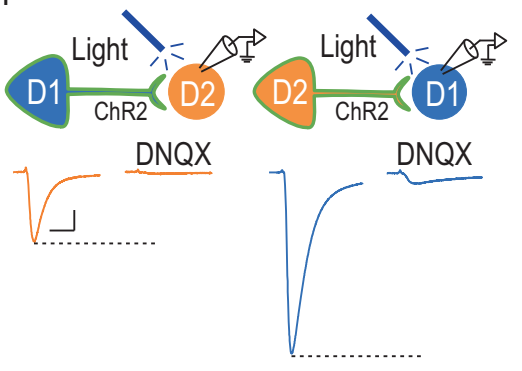

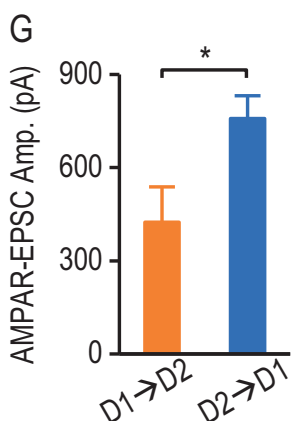

Fig. 1 Striatal glutamatergic synapses with different pre- and postsynaptic dopamine receptor expression exhibit distinct strengths. a A sagittal section from a D1-Cre;Ai32 mouse counterstained with NeuroTrace red (NT-Red) showed intense ChR2-EYFP expression in D1-MSNs, and in their projections to the internal segment of the globus pallidus (GPi) and the substantia nigra pars reticulata (SNr). Scale bar: $1 \mathrm{~mm}$. The boxed area is presented at higher magnification in the left-hand panels showing three striatal neurons stained with NT-Red (top); two neurons (arrowheads) expressing EYFP on the cell membrane (middle), and the merged image (yellow; bottom). Scale bar: $4 \mu \mathrm{m}$. $\mathbf{b} \mathrm{A}$ sagittal section from a D2-Cre;Ai32 mouse counterstained with NT-Red showed intense ChR2-EYFP expression in D2-MSNs and in their target, the external segment of the globus pallidus (GPe). Scale bar: $1 \mathrm{~mm}$. The left-hand panels showed two NT-Red-stained striatal neurons (top), one of which expressed EYFP (middle, arrowhead). Scale bar: $4 \mu \mathrm{m}$. c Sample traces of Sr${ }^{2+}$-induced asynchronous excitatory postsynaptic currents (aEPSCs) at synapses expressing pre- and postsynaptic D1Rs (D1 $\rightarrow$ D1), presynaptic D1Rs and postsynaptic D2Rs (D1 $\rightarrow$ D2), presynaptic D2Rs and postsynaptic D1Rs (D2 $\rightarrow$ D1), and pre- and postsynaptic D2Rs (D2 $\rightarrow$ D2). The EPSCs were evoked by 2-ms 470-nm light in the presence of 2.5 $\mathrm{mM} \mathrm{Sr}^{2+}$. d-e Bar graphs summarizing asynchronous EPSC frequency (d) and amplitude (e) after light stimulation of D1 $\rightarrow \mathrm{D} 1, \mathrm{D} 1 \rightarrow \mathrm{D} 2, \mathrm{D} 2 \rightarrow \mathrm{D} 1$, and D2 $\rightarrow \mathrm{D} 2$ synapses; ${ }^{* *} p<0.001$ versus the other three synapses (d), one-way ANOVA, post hoc SNK tests; $n=14$ neurons, 4 mice (D1 $\rightarrow$ D1); 12 neurons, 4 mice $(D 1 \rightarrow D 2) ; 13$ neurons, 4 mice $(D 2 \rightarrow D 1) ; 14$ neurons, 4 mice (D2 $\rightarrow$ D2). $f$ Top, schematic of whole-cell recordings of DMS D2MSNs in D1-Cre;Ai32 mice (left) and of DMS D1-MSNs from D2-Cre;Ai32 mice (right). Presynaptic neurons expressed ChR2 on the cell membrane (represented in green). Bottom, representative traces of light-evoked EPSCs in the absence and presence of DNQX (10 $\mu M)$ at $\mathrm{D} 1 \rightarrow \mathrm{D} 2$ (left) and D2 $\rightarrow \mathrm{D} 1$ (right) synapses. Scale bars: $20 \mathrm{~ms}, 80 \mathrm{pA}$. g Bar graphs comparing the amplitudes of AMPAR-EPSCs at D1 $\rightarrow$ D2 and $\mathrm{D} 2 \rightarrow \mathrm{D} 1$ synapses; ${ }^{*} p<0.05$, unpaired $t$ test; $n=6$ neurons, 5 mice $(\mathrm{D} 1 \rightarrow \mathrm{D} 2) ; 12$ neurons, 6 mice $(\mathrm{D} 2 \rightarrow \mathrm{D} 1)$

mechanisms underlying this effect have not been fully elucidated, they may involve the activation of presynaptic D2Rs on the glutamatergic terminal [24] and retrograde endocannabinoid (eCB) signaling via striatal postsynaptic D2Rs [25]. To investigate the effect of presynaptic D2Rs on cortical inputs, we took advantage of the absence of postsynaptic D2R expression at D2 $\rightarrow$ D1 synapses (Fig. 3a). We used paired-pulsed optical stimulation delivered at a frequency of $20 \mathrm{~Hz}[24,25]$ to activate D2R-expressing afferents, and recorded light-evoked responses in DMS D1-MSNs of D2-Cre;Ai32 mice before and during the bath application of a D2R agonist, quinpirole $(20 \mu \mathrm{M})$. We observed a significant inhibition of the first EPSC by quinpirole (Fig. 3b; 79.56 $\pm 3.78 \%$ of baseline; $\left.t_{(8)}=5.41, p<0.001\right)$. Moreover, this inhibition was accompanied by a significant increase in the paired-pulse ratio (PPR) (Fig. 3c; baseline, $0.27 \pm 0.03$; quinpirole, $0.39 \pm 0.04 ; t_{(8)}$ $=-6.46, p<0.001$ ), suggesting that quinpirole inhibited EPSCs by decreasing presynaptic glutamate release [26]. We next tested whether the quinpirole-mediated inhibition required eCB signaling by treating DMS slices with AM251, a cannabinoid type 1 receptor (CB1R) antagonist. With $3 \mu \mathrm{M} A M 251$ in the bath, quinpirole still inhibited EPSCs (Fig. 3d; EPSC $1=76.43 \pm 5.91 \%$ of baseline; $t_{(6)}=3.99, p<0.01$ ). AM251 also failed to prevent the quinpirole-induced increase in the PPR (Fig. 3e; baseline, $0.29 \pm$ 0.03 ; quinpirole, $\left.0.43 \pm 0.04 ; t_{(6)}=-8.75, p<0.001\right)$. These results indicate that presynaptic D2R signaling suppresses glutamatergic transmission in an eCB-independent manner. 
A

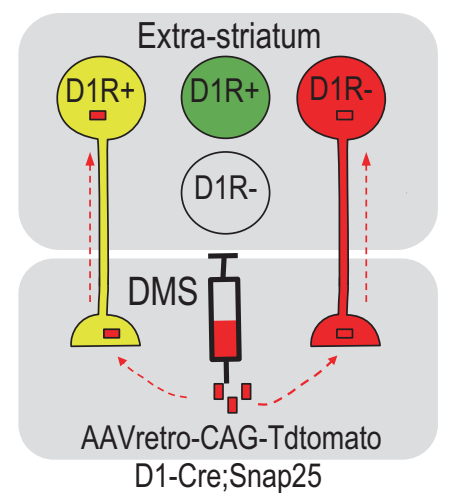

B

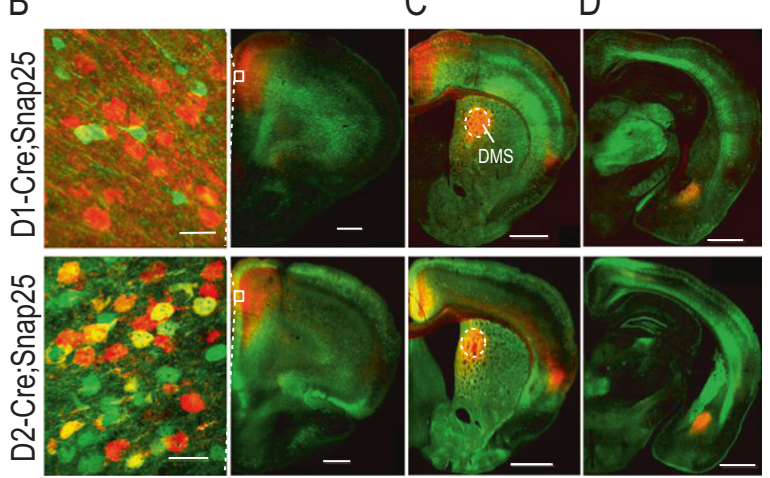

E

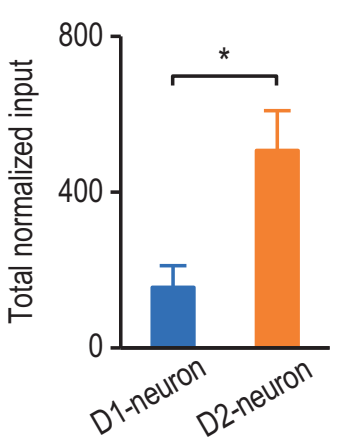

F

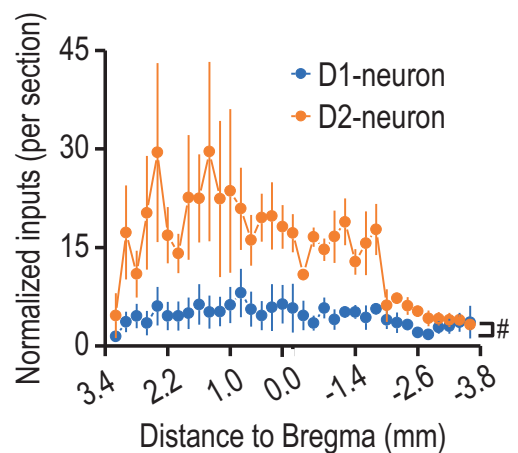

G

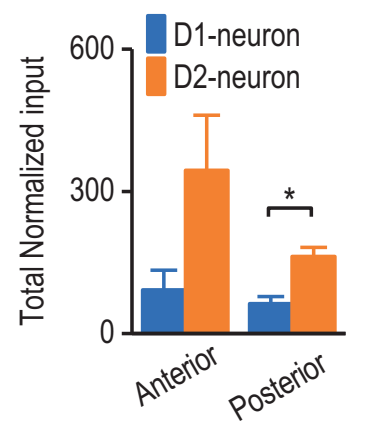

Fig. 2 DMS-projecting extra-striatal neurons preferentially express dopamine D2Rs versus D1Rs. a Illustration of the strategy used to target D1R-expressing $\left(D 1 R^{+}\right)$DMS-projecting extra-striatal neurons. We employed D1-Cre;Snap25 transgenic mice, where D1R-expressing neurons were labeled by GFP (green). The AAVretro-CAG-tdTomato was infused into the DMS of these mice, which labeled DMS-projecting neurons with a red fluorescent protein, tdTomato. Note that neurons co-expressing tdTomato and GFP were D1R-expressing DMS-projecting neurons (yellow). b-d Representative fluorescent images of D1R-expressing inputs (top) and D2R-expressing inputs (bottom) to the DMS from the cortex (b and c) and amygdala (d) following viral infusions in the DMS of D1-Cre;Snap25 and D2-Cre;Snap25 mice as indicated. Boxes (b, righthand panels) indicated the zone shown in the left-hand panels (b) at a higher magnification. Note that the number of yellow neurons was higher in D2-Cre;Snap25 mice than in D1Cre;Snap25 mice, although the numbers of red neurons were identical. Coronal sections were prepared 10 days after the viral infusion. Scale bar: $30 \mu \mathrm{m}$ (b, left); $500 \mu \mathrm{m}$ (b, right); $700 \mu \mathrm{m}$ (c); $800 \mu \mathrm{m}$ (d). e Bar graphs indicated that the total number of extra-striatal D2R-expressing neurons (D2-neuron) that projected to the DMS was significantly higher than that of extrastriatal D1R-expressing DMS-projecting neurons (D1-neuron). The total number of D1-neurons or D2-neurons was normalized to the red neurons in the $\mathrm{mPFC}^{*}{ }^{*} p<0.05$, unpaired $t$ test, $n=3$ mice per group. $\mathbf{f}$ Analysis of the distribution of D1-neurons and D2-neurons from the anterior to posterior (starting at $3.2 \mathrm{~mm}$ relative to Bregma and continuing in 200- $\mu \mathrm{m}$ steps) identified a significant difference between these neuronal types; ${ }^{p} p<0.05$, two-way RM ANOVA, $\mathrm{n}=102$ sections, 3 mice per group. $\mathbf{g}$ Bar graphs comparing the number of D1-neurons and D2-neurons in the anterior (starting at $3.2 \mathrm{~mm}$ and ending at $-0.2 \mathrm{~mm}$ relative to Bregma, where the anterior commercial crossed) and posterior (starting at $-0.2 \mathrm{~mm}$ and ending at $-3.6 \mathrm{~mm}$ relative to Bregma) regions; ${ }^{*} p<0.05$ (posterior), $p>0.05$ (anterior), unpaired $t$ test; $n=$ 3 mice per group

To determine the role of postsynaptic D2Rs on cortical inputs, we took advantage of the lack of presynaptic D2R expression at the D1 $\rightarrow$ D2 synapse (Fig. 3f). We recorded D2-MSNs of D1-Cre; Ai32 mice, performed the same experiment as in Fig. 3a-e, and observed a similar inhibitory effect of quinpirole. The first EPSC amplitude was significantly reduced after quinpirole application (Fig. $3 g ; 72.18 \pm 2.38 \%$ of baseline; $t_{(8)}=11.67, p<0.001$ ), and PPR was significantly increased (Fig. $3 \mathrm{~h}$; baseline, $0.26 \pm 0.04$; quinpirole, $\left.0.34 \pm 0.05 ; t_{(8)}=-4.73, p<0.01\right)$. We next tested whether postsynaptic D2R-mediated inhibition required retrograde eCB signaling. In the presence of AM251, quinpirole failed to inhibit $\mathrm{D} 1 \rightarrow \mathrm{D} 2$ EPSCs (Fig. 3i; $99.7 \pm 4.43 \%$ of baseline; $t_{(8)}=0.07, p>$ 0.05 ) or to change PPR (Fig. 3j; baseline, $0.27 \pm 0.05$; quinpirole, $\left.0.28 \pm 0.05 ; t_{(8)}=-1.64, p>0.05\right)$, indicating that CB1R activation is necessary for postsynaptic D2R-mediated synaptic depression. Taken together, these results suggest that D2R-mediated inhibition of glutamatergic transmission in the DMS involves distinct pre- and postsynaptic mechanisms, and that postsynaptic D2Rmediated inhibition requires eCB signaling.
Excessive alcohol consumption causes a long-lasting potentiation of glutamatergic transmission at the DMS D2 $\rightarrow$ D1 synapse Altered dopaminergic transmission is the common mechanism underlying drug and alcohol abuse. To investigate how excessive alcohol intake alters $\mathrm{D} 2 \rightarrow \mathrm{D} 1$ and $\mathrm{D} 1 \rightarrow \mathrm{D} 2$ synaptic transmission, D2-Cre;Ai32 and D1-Cre;Ai32 mice were trained to consume $20 \%$ alcohol for 8 weeks using the intermittent-access two-bottle choice drinking procedure $[2,5,16,17]$. As shown in Table S1, these mice consumed high levels of alcohol, and the drinking levels were identical between the two mouse lines (Table S1; $q=$ $0.58, p>0.05)$. The water control mice underwent the same treatment, but without alcohol exposure. Twenty-four hours after the last alcohol exposure, we prepared DMS slices and measured light-evoked AMPAR-mediated EPSCs in MSNs. We found that the amplitude of D2 $\rightarrow$ D1 EPSCs was significantly higher in the alcohol-drinking group than in the water controls (Fig. 4a; $F_{(1,84)}$ $=10.01, p<0.01)$. Additionally, excessive alcohol intake produced a long-lasting ( 9 days) increase in AMPAR-EPSCs (Fig. $4 a ; F_{(1,100)}=$ 7.93, $p<0.01)$. In contrast, the amplitude of D1 $\rightarrow$ D2 EPSCs was 
A

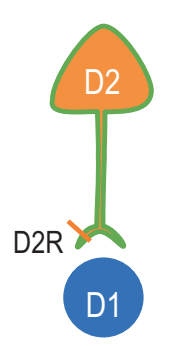

B

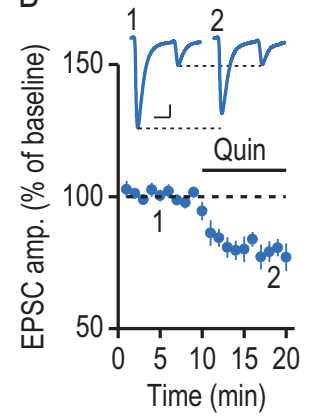

F

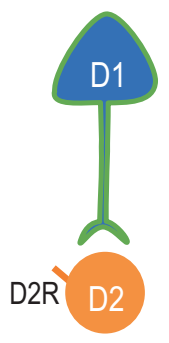

G

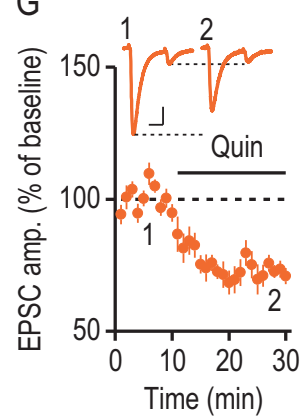

C

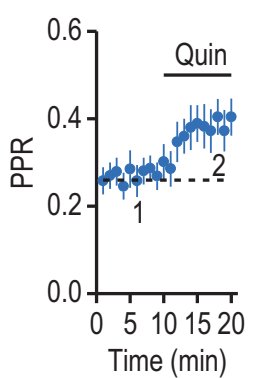

$\mathrm{H}$

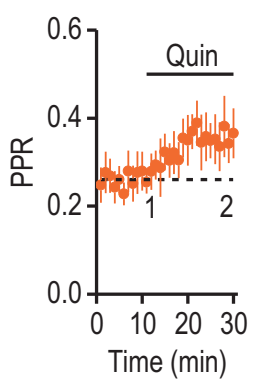

D

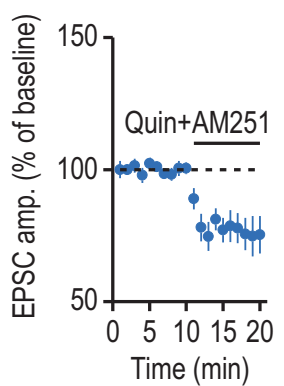

I

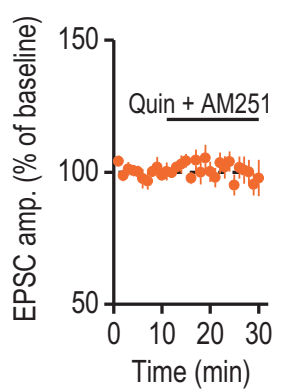

E

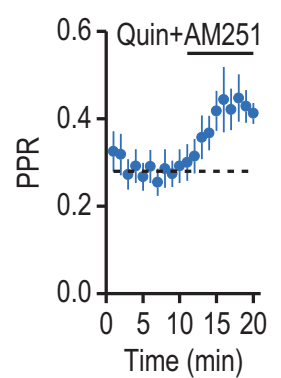

J

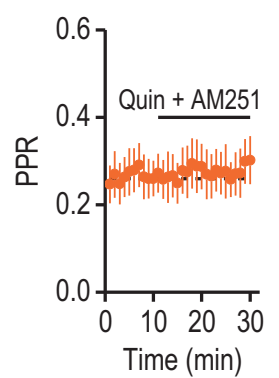

Fig. 3 Suppression of glutamatergic transmission at different DMS synapses via pre- and postsynaptic D2Rs. a Illustration indicating wholecell recordings of D2 $\rightarrow$ D1 transmission in D2-Cre;Ai32 mice. D2Rs were selectively expressed at presynaptic terminals, but not in postsynaptic D1-MSNs. b Activation of presynaptic D2Rs by bath application of quinpirole (Quin, $20 \mu \mathrm{M}$ ) suppressed the first of two EPSCs induced by paired-pulse (50-ms interval) stimulation of D2R-expressing inputs; ${ }^{*} p<0.05$, paired $t$ test; $n=9$ slices, 7 mice. The inset sample traces indicated the light-evoked paired-pulse EPSCs in the absence (1) and presence (2) of quinpirole. Scale bars: $20 \mathrm{ms,} 100$ pA. c Time-course of the PPR (the second EPSC amplitude divided by the first EPSC amplitude) before and during quinpirole application; ${ }^{*} p<0.05$, paired $\mathrm{t}$ test; $\mathrm{n}=$ 9 slices, 7 mice. d Bath application of AM251 did not affect the quinpirole-induced suppression of the first EPSC; ${ }^{*} p<0.05$, paired $t$ test; $n=$ 7 slices, 6 mice. e Quinpirole-induced increases in the PPR were also observed in the presence of AM251; ${ }^{*} p<0.05$, paired $t$ test; $n=7$ slices, 6 mice. f Illustration showing whole-cell recordings of D1 $\rightarrow$ D2 transmission in D1-Cre;Ai32 mice. D2Rs were selectively expressed in postsynaptic D2-MSNs, but not at presynaptic terminals. g Activation of postsynaptic D2Rs by quinpirole suppressed the first of two EPSCs; ${ }^{*} p<0.05$, paired $t$ test; $n=9$ slices, 4 mice. The inset sample traces indicate the two light-evoked EPSCs in the absence (1) and presence (2) of quinpirole. Scale bars: $20 \mathrm{~ms}, 50 \mathrm{pA}$. $\mathbf{h}$ Time-course of the PPR before and during quinpirole application; ${ }^{*} p<0.05$, paired $t$ test; $n=9$ slices, 4 mice. i AM251 bath application abolished quinpirole-induced synaptic suppression; $p>0.05$, paired $t$ test; $n=9$ slices, 5 mice. j AM251 abolished quinpirole-induced increases in the PPR; $p>0.05$, paired $t$ test; $n=9$ slices, 5 mice

identical in both alcohol and water groups (Fig. 4b; $F_{(1,116)}=0.57$, $p>0.05)$. Interestingly, we also discovered that excessive alcohol intake caused a long-lasting decrease in PPR at the D2 $\rightarrow$ D1 synapse (Fig. 4c; 24-h withdrawal, $t_{(21)}=2.432, p<0.05$; 9-d withdrawal, $\left.t_{(26)}=2.648, p<0.05\right)$; this effect was not observed at the D1 $\rightarrow \mathrm{D} 2$ connection (Fig. $4 \mathrm{~d} ; t_{(22)}=-0.318, p>0.05$ ). Since the PPR is inversely correlated with transmitter release probability [26], this reduction in PPR indicated that the alcohol-induced enhancement of D2 $\rightarrow \mathrm{D} 1$ connectivity was mediated, at least in part, by an increased glutamate release. Collectively, these data demonstrate that excessive alcohol consumption persistently enhances glutamatergic transmission at the D2 $\rightarrow$ D1 synapse of the DMS.

In the striatum, D2Rs are also expressed in cholinergic interneurons (CINs) and dopaminergic fibers [6, 27], leading to ChR2 expression by these cells in D2-Cre;Ai32 mice. Glutamate [28], acetylcholine, and dopamine might be released during light stimulation. However, we found that glutamatergic transmission from CINs to MSNs represented a small proportion of the overall $\mathrm{D} 2 \rightarrow \mathrm{D} 1$ transmission (Figure S3A-S3C), suggesting that most D2 $\rightarrow$ D1 synapses involved extra-striatal neurons. The lightevoked release of acetylcholine and dopamine was unlikely to influence the observed glutamatergic D2 $\rightarrow$ D1 transmission (Figure S3D-S3E).
The strong mPFC D2R-expressing input onto DMS D1-MSNs is potentiated by excessive alcohol consumption

The data shown in Fig. 1 indicated that glutamatergic transmission from brain-wide D2R-expressing afferents onto D1-MSNs was stronger than that observed at the other tested connections. However, we also wished to investigate the strength of D2Rexpressing afferents from the mPFC onto this neuronal type. Additionally, we recently found that excessive alcohol consumption increased AMPAR activity at the MPFC input to the DMS [14]. To access the D2R-expressing mPFC input, a Cre-dependent Chronos-expressing AAV [29] was infused into the MPFC of D2Cre;Ai14 mice (Fig. 5a), which express Cre and tdTomato in D2MSNs and in mPFC D2R-expressing neurons $[2,5]$. The expression of Chronos was indicated by the presence of GFP, which was restricted selectively to D2R-expressing (red) MPFC neurons (Fig. 5b). Infusion of AAV-Flex-Chronos-GFP caused intense green fluorescent labeling of mPFC D2R-expressing afferents in the DMS (Fig. 5c). DMS D2-MSNs were identified by the red fluorescence of tdTomato (Fig. 5c) and any non-fluorescent striatal MSNs were therefore considered as putative D1-MSNs. Whole-cell recordings were performed from these two types of MSN. Similar to the $\mathrm{D} 2 \rightarrow \mathrm{D} 1$ connection in D2-Cre;Ai32 mice, bath application of DNQX $(10 \mu \mathrm{M})$ and an NMDAR antagonist (APV, $50 \mu \mathrm{M})$ completely abolished light-evoked activity at the mPFC D2R-expressing inputs 
A

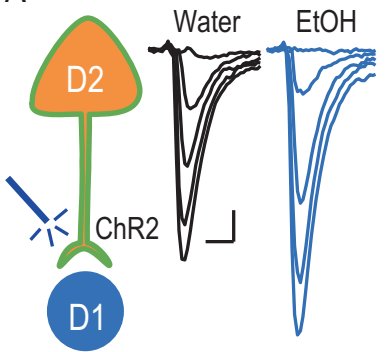

B

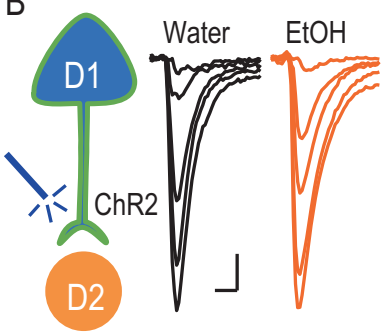

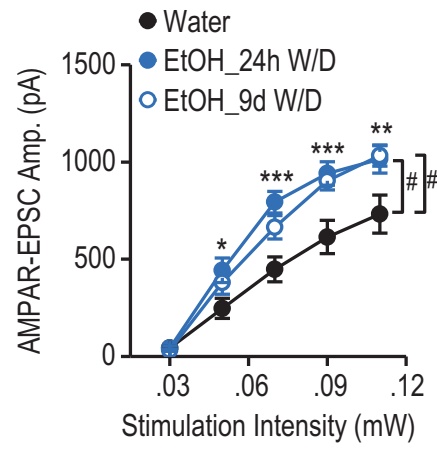
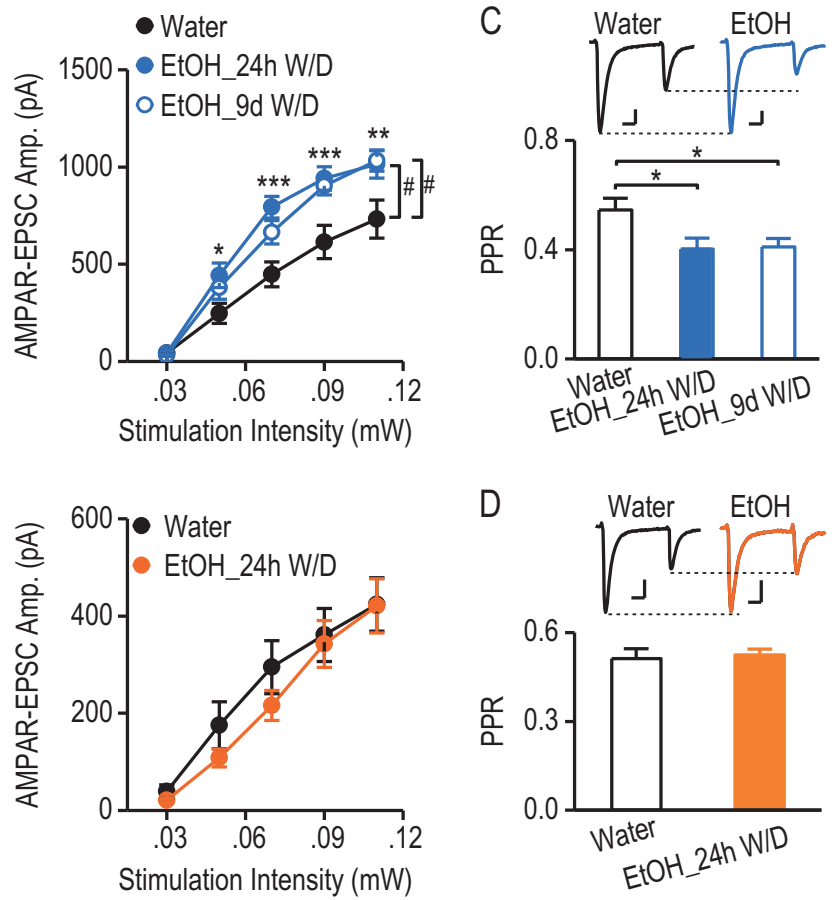

D
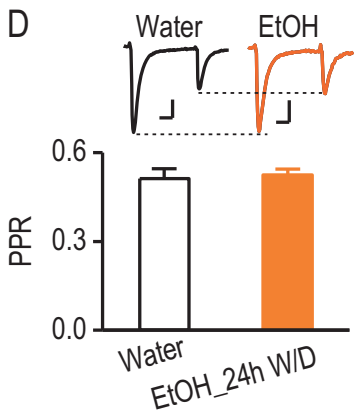

Fig. 4 Excessive alcohol consumption causes a long-lasting potentiation of glutamatergic D2 $\rightarrow$ D1 transmission. a Excessive alcohol intake persistently increased the amplitude of AMPAR-mediated EPSCs at the D2 $\rightarrow$ D1 connection. Whole-cell recordings were performed $24 \mathrm{~h}$ or $9 \mathrm{~d}$ after the last alcohol exposure. Left, illustration indicating that optical stimulation of ChR2-expressing D2R-expressing inputs in D2-Cre;Ai32 mice activated D2 $\rightarrow$ D1 glutamatergic transmission. Middle, the representative traces of AMPAR-EPSCs evoked by a range of optical stimulation intensities in slices from alcohol-drinking mice (EtOH) and water controls. Scale bar: 10 ms, 100 pA. Right, input-output curves of optical AMPAR-EPSCs at the D2 $\rightarrow$ D1 synapse from mice exposed to alcohol with $24 \mathrm{~h}$ withdrawal (EtOH_24h W/D), alcohol with $9 \mathrm{~d}$ withdrawal (EtOH_9d W/D), or water only; ${ }^{\#} p<0.01$, two-way RM ANOVA; ${ }^{*} p<0.05 ;{ }^{* *} p<0.01 ;{ }^{* * *} p<0.001$ EtOH_24h W/D versus water group at the same stimulation intensities; post hoc SNK test; $n=12$ neurons, 6 mice (Water); 11 neurons, 5 mice (EtOH_24h W/D); and 15 neurons, 5 mice $\left(E t O H \_9 d\right.$ W/D). b Excessive alcohol intake did not alter the amplitude of D1 $\rightarrow$ D2 AMPAR-EPSCs. Left, illustration indicating that light stimulation of ChR2 expressed at presynaptic terminals of extra-striatal D1R-expressing neurons in D1-Cre;Ai32 mice triggered D1 $\rightarrow$ D2 glutamatergic transmission. Middle, sample traces of AMPAR-EPSCs evoked by the indicated optical stimulation intensities in slices from alcoholdrinking mice and water controls. Scale bar: $10 \mathrm{~ms}, 80 \mathrm{pA}$. Right, the input-output curves of D1 $\rightarrow$ D2 AMPAR-EPSCs from the indicated study groups; $p>0.05$, versus same intensities in the water group, two-way RM ANOVA; $\mathrm{n}=15$ neurons, 7 mice (Water); 16 neurons, 7 mice (EtOH). c Excessive alcohol intake increased the glutamate release probability at D2 $\rightarrow$ D1 synapses. PPRs were calculated by comparing paired AMPAREPSCs induced by two optical stimuli, delivered at a 100-ms interval. Bar graph summarizing PPRs in the indicated groups; ${ }^{*} p<0.05$, unpaired t test; $n=12$ neurons, 4 mice (Water); 11 neurons, 6 mice (EtOH_24h withdrawal); 16 neurons, 5 mice (EtOH_9d withdrawal). The inset shows paired AMPAR responses in the water and alcohol groups. Scale bar: $20 \mathrm{~ms}, 50 \mathrm{pA}$. d Excessive alcohol intake did not change the glutamate release probability at $\mathrm{D} 1 \rightarrow \mathrm{D} 2$ synapses. Bar graph comparing the corresponding mean PPRs in the indicated groups; $p>0.05$, unpaired $t$ test; $n=12$ neurons, 5 mice per group. The inset shows paired AMPAR responses in the water and alcohol groups. Scale bar: $20 \mathrm{~ms}, 50 \mathrm{pA}$

onto both DMS D1-MSNs (Fig. 5d) and D2-MSNs (Fig. 5e), suggesting that they were glutamatergic. To compare the glutamatergic strength of D2 $\rightarrow \mathrm{D} 1$ and $\mathrm{D} 2 \rightarrow \mathrm{D} 2$ connections, we measured light-evoked AMPAR-EPSCs in D2-Cre;Ai14 mice. We found that the amplitudes of AMPAR-EPSCs were significantly higher in the $D 2 \rightarrow D 1$ group than in the $D 2 \rightarrow D 2$ group (Fig. $5 f$; $\left.F_{(1,112)}=4.95, p<0.05\right)$. These results indicate that mPFC D2Rexpressing inputs distinctly control D1-MSNs and D2-MSNs, with a stronger glutamatergic connection between mPFC D2Rexpressing neurons and DMS D1-MSNs.

Lastly, we explored the effect of excessive alcohol intake on AMPAR-mediated EPSCs at mPFC D2R-expressing inputs onto DMS D1- or D2-MSNs. D2-Cre;Ai14 mice with mPFC infusion of AAV-Flex-Chronos-GFP were trained to consume high levels of alcohol (Table S1), as described above. Twenty-four hours after the last alcohol exposure, we prepared DMS slices and measured light-evoked EPSCs in DMS D1-MSNs and D2-MSNs. We found that excessive alcohol intake selectively potentiated AMPAR-EPSCs at D2R-expressing mPFC inputs onto DMS D1-MSNs (Fig. $5 \mathrm{~g} ; F_{(1,112)}$ $=8.36, p<0.01)$. In contrast, excessive alcohol intake did not alter EPSCs at D2R-expressing mPFC inputs onto DMS D2-MSNs (Fig. 5h; $\left.F_{(1,112)}=0.46, p>0.05\right)$. We also investigated whether excessive alcohol intake altered presynaptic glutamate release. A marginally lower PPR was observed at the D2 $\rightarrow$ D1 synapse of the mPFC-DMS pathway in alcohol-drinking mice, as compared with the water controls (Fig. $5 i_{;} t_{(28)}=1.77, p=0.087$ ). However, the PPR at the D2 $\rightarrow$ D2 synapse did not differ between the alcohol and water groups (Fig. $5 \mathrm{i} ; t_{(28)}=-0.22, p>0.05$ ). These data suggest that excessive alcohol intake may increase the probability of glutamate release selectively at the mPFC D2R-expressing afferents onto D1-MSNs. In summary, these results suggest that excessive alcohol intake enhances AMPAR-mediated glutamate transmission selectively at the D2 $\rightarrow \mathrm{D} 1$ synapse within the mPFCDMS pathway.

\section{DISCUSSION}

The present study dissected glutamatergic afferents to the striatum based on their dopamine receptor expression, and revealed that the glutamatergic connection between presynaptic D2R-expressing afferents and DMS D1-MSNs was stronger than that of the other three tested connections. This uneven synaptic strength may result in differential dopaminergic modulation of glutamatergic transmission. Additionally, we discovered that 
postsynaptic D2R-mediated suppression of glutamatergic transmission required eCB signaling, whereas presynaptic D2Rs mediated eCB-independent suppression. Our results contribute to the clarification of previous controversial findings relating to the role of the D2R in inhibitory presynaptic filtering of cortical inputs. Lastly, we found that excessive alcohol intake selectively strengthened glutamatergic transmission at the D2 $\rightarrow$ D1 synapse, suggesting that this circuit may play a critical role in the pathogenesis of alcohol use disorder.

Glutamatergic connectivity at DMS synapses with distinct pre- and postsynaptic dopamine receptors

Dopamine is a key player in brain reward circuitry [1]. Investigations of cortical inputs onto MSNs have demonstrated that a major role of dopamine is to promote transient shifts in the balance between the direct and indirect striatal pathways of the basal ganglia during reward-driven behaviors $[1,30]$. Modulation of presynaptic filtering by dopamine may be critical in the determination of which cortical and thalamic signals are transduced by the appropriate excitatory striatal synapses during learning. Since D1Rs and D2Rs are major dopamine receptors in the corticostriatal pathway, we employed D1-Cre;Ai32 and D2-Cre; Ai32 mice to dissect the circuitry into at least four types of synapse: $\mathrm{D} 1 \rightarrow \mathrm{D} 1, \mathrm{D} 1 \rightarrow \mathrm{D} 2, \mathrm{D} 2 \rightarrow \mathrm{D} 1$, and $\mathrm{D} 2 \rightarrow \mathrm{D} 2$.
In both brain-wide and MPFC studies, we found that glutamatergic D2 $\rightarrow$ D1 transmission was stronger than that observed at the tested connection. It is known that in the dorsal striatum, D2Rs are more prevalent than D1Rs presynaptically at corticostriatal projections $[1,7,8]$. This may explain our findings indicating that D2R-expressing inputs were stronger than D1Rexpressing afferents in the DMS. However, our observation that the $D 2 \rightarrow D 1$ connection was stronger than the D2 $\rightarrow$ D2 synapse indicated a difference at the postsynaptic site. Midbrain dopamine neurons predominantly fire at a low frequency, and such tonic activity builds up the basal level of dopamine in the striatum [1]. This tonic dopamine inhibits D2-MSNs via postsynaptic D2Rs. When the glutamatergic and dopaminergic inputs are activated at high frequency, eCB is preferentially generated in the D2-MSNs; this eCB suppresses presynaptic glutamate release onto D2-MSNs [1]. Additionally, at the behavioral level, the striatal circuit plays a dual role in the modulation of action initiated by the cortical inputs, by reinforcing the currently selected action via the direct pathway and suppressing potentially conflicting actions via the indirect pathway [31]. Therefore, the intrinsically stronger D2 $\rightarrow$ D1 circuit (as compared with the D2 $\rightarrow$ D2 synapse) in the DMS may provide a mechanism for processing information through dopamine-dependent activation of the specific direct pathway in order to procure a reward. The current study only investigated
A

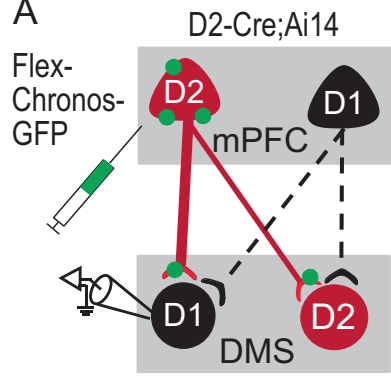

C

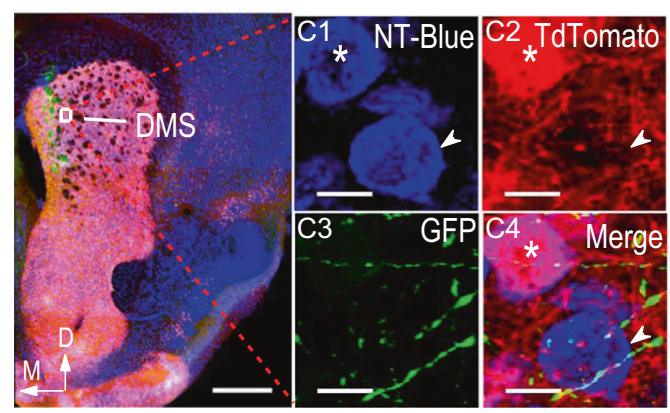

F

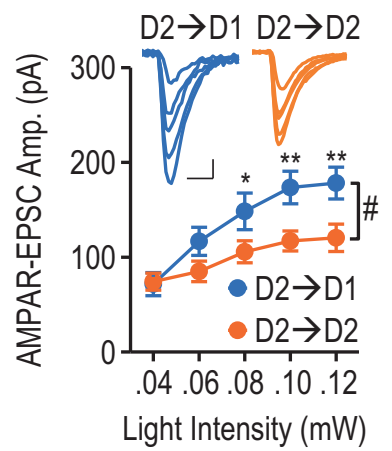

B
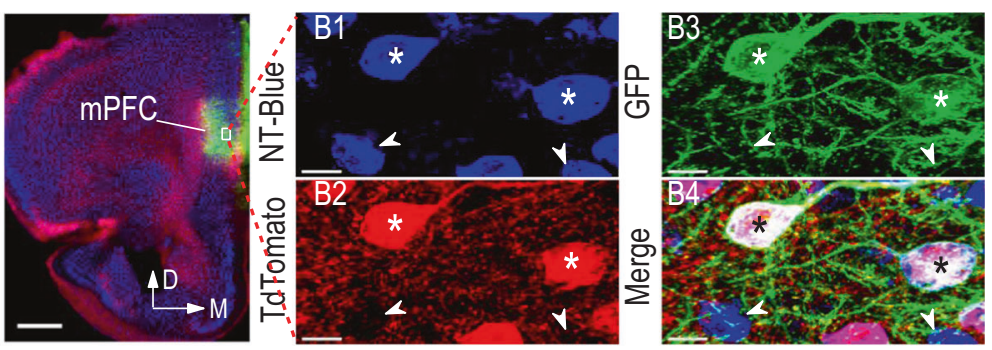

D
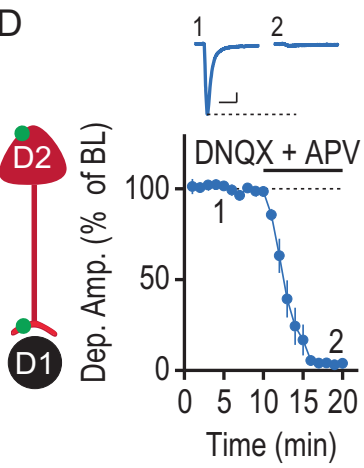

E
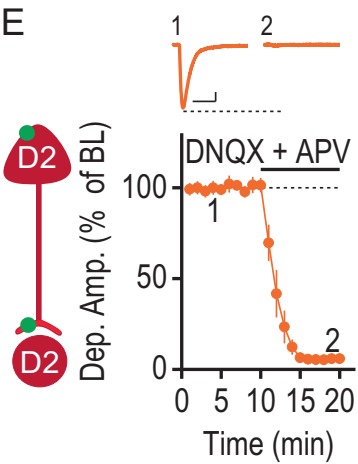

$\mathrm{H}$

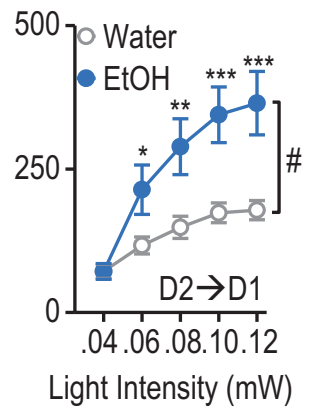

I
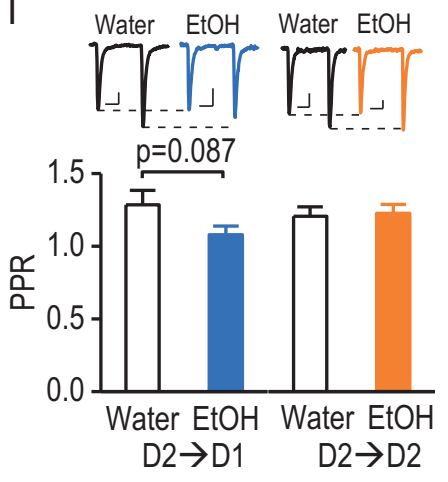
Fig. 5 The strong mPFC D2R-expressing input onto DMS D1-MSNs is potentiated by excessive alcohol consumption. a Illustration depicting the infusion of AAV-Flex-Chronos-GFP into the mPFC of D2-Cre;Ai14 mice, resulting in selective expression of Chronos in mPFC D2Rexpressing neurons, but not D1R-expressing neurons. The expressed Chronos (green) was trafficked down to the mPFC terminals within the DMS. Optical stimulation exclusively activated glutamatergic transmission from mPFC D2R-expressing inputs onto DMS D1-MSNs (D2 $\rightarrow$ D1) or D2-MSNs (D2 $\rightarrow$ D2). D2-MSNs were identified by their expression of tdTomato. The tdTomato-negative striatal neurons were considered as putative D1-MSNs. b Representative images of the MPFC infusion site. Coronal sections were prepared 8 weeks after the viral infusion. The section was counterstained with NeuroTrace blue (NT-Blue). Infusion of AAV-Flex-Chronos-GFP caused intense green fluorescent labeling at the injection site (left). Scale bar: $500 \mu \mathrm{m}$. The boxed area is presented at higher magnification in the right-hand panels. All mPFC neurons were stained with NT-Blue (B1). D2-neurons expressed tdTomato (red), and the arrows indicate D2R-negative cells (B2). AAV-Flex-Chronos-GFP was selectively expressed in D2R-expressing neurons (B3, stars). Stars indicate the Chronos-expressing tdTomato-positive neurons. Arrows indicate that tdTomato-negative cells did not express Chronos. Scale bar: $10 \mu \mathrm{m}$. c Sample images of Chronos-expressing mPFC fibers in the DMS (left). The striatal neurons from the indicated box are shown on the right. All neurons were blue-stained (C1). D2-MSNs (stars) expressed red tdTomato and putative D1-MSNs are indicated by arrows (C2). Green mPFC afferents innervated striatal neurons (C3). The merged image (C4) shows green fibers surrounded by both D2-MSNs (red and blue) and putative D1-MSNs (blue). Scale bar: $500 \mu \mathrm{m}$ (left), and $7 \mu \mathrm{m}$ (right). d Verification of glutamatergic transmission at D2 $\rightarrow$ D1 synapses. Illustration indicating the whole-cell recordings performed on D1-MSNs. Synaptic transmission was triggered by optical stimulation of Chronos-expressing D2R-expressing inputs from the mPFC. The light-evoked responses were completely abolished by bath application of the AMPAR antagonist, DNQX $(10 \mu M)$, and the NMDAR antagonist, APV $(50 \mu M)$; DNQX + APV: $4.00 \pm 0.73 \%$ of baseline, $t_{(9)}=131.27, p<0.05$, paired $t$ test; $n=10$ slices, 8 mice. The inset sample traces indicate the lightevoked responses in D1-MSNs at baseline (1) and after infusion of DNQX and APV (2). Scale bars: 20 ms, 40 pA. e Verification of glutamatergic transmission at D2 $\rightarrow$ D2 synapses. Illustration showing that light-evoked responses were recorded from D2-MSNs. Bath application of DNQX and APV completely blocked these light-evoked responses; DNQX + APV: $5.43 \pm 0.89 \%$ of baseline, $t_{(9)}=106.82, p<0.05$, paired $t$ test; $n=$ 10 slices, 8 mice. The inset sample traces indicate the light-evoked responses in D2-MSNs before (1) and after (2) infusion of DNQX and APV. Scale bars: $20 \mathrm{~ms}, 25 \mathrm{pA}$. Note that there was no ChR2 expression in the D2-MSNs of D2-Cre;Ai14 mice. Therefore, blockade of glutamatergic transmission completely abolished the light-evoked response, in contrast to the partial D2 $\rightarrow$ D2 inhibition in D2-Cre;Ai32 mice. f AMPARmediated EPSCs were significantly larger at the D2 $\rightarrow$ D1 connection than at the D2 $\rightarrow$ D2 synapse. Left, Representative traces of the responses evoked in DMS D1- and D2-MSNs by a range of stimulation intensities. Scale bars: $10 \mathrm{~ms}, 30 \mathrm{pA}$. Right, The corresponding input-output curves; ${ }^{\#} p<0.05 ;{ }^{*} p<0.05 ;{ }^{* *} p<0.01$ versus water group at the same stimulation intensities, two-way RM ANOVA followed by SNK test; $n=15$ neurons, 5 mice per group. $g$ Excessive alcohol intake significantly increased the amplitude of AMPAR-mediated EPSCs from the mPFC D2inputs onto D1-MSNs. The input-output curves of D2 $\rightarrow$ D1 AMPAR-EPSCs were measured at a range of stimulation intensities in the water and alcohol (EtOH)-drinking groups; ${ }^{\#} p<0.05$, two-way RM ANOVA; ${ }^{*} p<0.05$; ${ }^{* *} p<0.01$; ${ }^{* * *} p<0.001$ versus water group at the same stimulation intensities, post hoc SNK test; $n=15$ neurons, 5 mice per group. $\mathbf{h}$ Excessive alcohol intake did not alter the AMPAR-mediated D2-MSN response to mPFC D2-inputs. The input-output curves of D2 $\rightarrow$ D2 AMPAR-EPSC were measured at the indicated stimulation intensities; $p>$ 0.05 , two-way RM ANOVA; $n=15$ neurons, 5 mice per group. $\mathbf{i}$ Excessive alcohol consumption caused a marginal reduction in PPR at the $\mathrm{D} 2 \rightarrow \mathrm{D} 1$ connection, but not at the D2 $\rightarrow \mathrm{D} 2$ connection. Bar graphs comparing PPRs in the indicated groups; $p=0.087$, unpaired $t$ test; $n=15$ neurons, 5 mice per group (D2 $\rightarrow \mathrm{D} 1$ ); $p>0.05$, unpaired $t$ test; $n=15$ neurons, 5 mice per group (D2 $\rightarrow$ D2). Inset, representative AMPAR-EPSC traces induced by two optical stimuli delivered at a 100-ms interval in the water and EtOH groups. Scale bars: $30 \mathrm{~ms}, 30 \mathrm{pA}$ (D2 $\rightarrow$ D1); $30 \mathrm{~ms}$, $15 \mathrm{pA}(\mathrm{D} 2 \rightarrow \mathrm{D} 2)$

four types of dopamine receptor-based pathways. Although neurons expressing D1Rs or D2Rs were highly segregated in the cortical and striatal regions, some co-localization was observed $[2,5]$. Co-expressed D1Rs and D2Rs can form heterodimers, which contribute to addiction and schizophrenia [32]. Due to the limitations of our transgenic animals, the influence of this subpopulation on the corticostriatal pathway remains to be determined.

\section{Alcohol-evoked circuit-specific plasticity}

Accumulating evidence suggests that exposure to addictive substances, including alcohol, potentiates AMPAR-mediated responses in D1-MSNs, but not D2-MSNs [5, 16, 33]. Our current findings were consistent with this, with no alcohol-related changes observed in D2-MSNs. Importantly, D1-MSNs with inputs from extra-striatal neurons expressing D2Rs, such as mPFC D2neurons, did exhibit marked alterations after repeated alcohol exposure. It is not fully clear why chronic alcohol exposure selectively potentiates the glutamatergic connectivity of D2 $\rightarrow$ D1, while not affecting other synapses. Our observation of a decreased PPR indicated that this potentiation was mediated, at least in part, by an increase in presynaptic glutamate release. Presynaptic terminals are known to express D2Rs [8], and their activation by tonic dopamine is reported to inhibit glutamate release $[25,34]$. The basal dopamine level is reported to decline gradually after a prolonged period of excessive alcohol drinking and withdrawal cycles $[35,36]$. The $D 2 R$ is a high-affinity dopamine receptor that is classically assumed to inhibit neuronal activity via $\mathrm{Ga}_{\mathrm{i}}$ protein or $\beta$-arrestin [37]. Activation of $\mathrm{D} 2 \mathrm{R}$ also blocks excitatory currents by recruiting Akt-GSK3 signaling [38]. The decreased basal dopamine level may reduce D2R-mediated suppression of $\mathrm{D} 2 \rightarrow \mathrm{D} 1$ signaling, which may consequently enhance glutamatergic transmission within this pathway and thus contribute to excessive drinking behavior.

\section{D1-Cre and D2-Cre mouse lines}

To achieve specific stimulation of extra-striatal inputs, we used D1Cre and D2-Cre lines crossed with an optogenetic reporter mouse line. One limitation of this approach is that the representation of D1R-expressing neurons in the D1-Cre mouse and of D2Rexpressing neurons in D2-Cre mouse might not be equally reliable. However, we compared the Cre efficiency of D1-Cre and D2-Cre mice, and the results suggested that these mouse lines showed equivalent levels of dopamine receptor expression. Thus, the observed difference in glutamatergic strength is highly likely to relate to intrinsic differences in the pathways, rather than to Cre line efficiency differences in D1-Cre and D2-Cre mice.

In conclusion, we demonstrated that inputs to the DMS can be classified in a cell type-specific manner and that the strengths of their glutamatergic connections with two types of MSN were not identical. The connection between corticostriatal D2R-expressing inputs and D1-MSNs was stronger than that of the other three tested connections, and was selectively potentiated by excessive alcohol intake. The elucidation of this alcohol-evoked circuit-specific plasticity could contribute to the identification of new neuronal therapeutic targets for the treatment of alcohol use disorder.

\section{FUNDING AND DISCLOSURE}

This research was funded by R01AA021505 (JW), U01AA025932 (JW), and the Texas Research Society on Alcoholism (JL). 


\section{ADDITIONAL INFORMATION}

Supplementary Information accompanies this paper at (https://doi.org/10.1038/ s41386-019-0332-9)

Conflict of interests: The authors declare no competing interests.

Publisher's note: Springer Nature remains neutral with regard to jurisdictional claims in published maps and institutional affiliations.

\section{REFERENCES}

1. Bamford NS, Wightman RM, Sulzer D. Dopamine's effects on corticostriatal synapses during reward-based behaviors. Neuron. 2018;97:494-510.

2. Wei X, Ma T, Cheng Y, Huang CCY, Wang X, Lu J, et al. Dopamine D1 or D2 receptor-expressing neurons in the central nervous system. Addict Biol. 2018;23:569-84

3. Beaulieu JM, Gainetdinov RR. The physiology, signaling, and pharmacology of dopamine receptors. Pharmacol Rev. 2011;63:182-217.

4. Lovinger DM. Neurotransmitter roles in synaptic modulation, plasticity and learning in the dorsal striatum. Neuropharmacology. 2010;58:951-61.

5. Cheng Y, Huang CC, Ma T, Wei X, Wang X, Lu J, et al. Distinct synaptic strengthening of the striatal direct and indirect pathways drives alcohol consumption. Biol Psychiatry. 2017;81:918-29.

6. Kreitzer AC, Malenka RC. Striatal plasticity and basal ganglia circuit function. Neuron. 2008;60:543-54.

7. Dumartin B, Doudnikoff E, Gonon F, Bloch B. Differences in ultrastructural localization of dopaminergic D1 receptors between dorsal striatum and nucleus accumbens in the rat. Neurosci Lett. 2007;419:273-7.

8. Wang H, Pickel VM. Dopamine D2 receptors are present in prefrontal cortical afferents and their targets in patches of the rat caudate-putamen nucleus. J Comp Neurol. 2002;442:392-404.

9. Luscher C, Malenka RC. Drug-evoked synaptic plasticity in addiction: from molecular changes to circuit remodeling. Neuron. 2011;69:650-63.

10. Koob GF, Volkow ND. Neurocircuitry of addiction. Neuropsychopharmacology. 2010;35:217-38.

11. Corbit LH, Leung BK, Balleine BW. The role of the amygdala-striatal pathway in the acquisition and performance of goal-directed instrumental actions. J Neurosci. 2013;33:17682-90.

12. Balleine BW, O'Doherty JP. Human and rodent homologies in action control: corticostriatal determinants of goal-directed and habitual action. Neuropsychopharmacology. 2010;35:48-69.

13. Ma YY, Lee BR, Wang $X$, Guo $C$, Liu L, Cui R, et al. Bidirectional modulation of incubation of cocaine craving by silent synapse-based remodeling of prefrontal cortex to accumbens projections. Neuron. 2014;83:1453-67.

14. Ma T, Barbee B, Wang X, Wang J. Alcohol induces input-specific aberrant synaptic plasticity in the rat dorsomedial striatum. Neuropharmacology. 2017;123:46-54.

15. Ma T, Cheng Y, Roltsch Hellard E, Wang X, Lu J, Gao X, et al. Bidirectional and long-lasting control of alcohol-seeking behavior by corticostriatal LTP and LTD. Nat Neurosci. 2018;21:373-83.

16. Wang J, Cheng $Y$, Wang X, Roltsch Hellard E, Ma T, Gil H, et al Alcohol elicits functional and structural plasticity selectively in dopamine D1 receptor-expressing neurons of the dorsomedial striatum. J Neurosci. 2015;35:11634-43.

17. Cheng Y, Wang X, Wei X, Xie X, Melo S, Miranda RC, et al. Prenatal exposure to alcohol induces functional and structural plasticity in dopamine D1 receptorexpressing neurons of the dorsomedial striatum. Alcohol Clin Exp Res. 2018:42:1493-502.
18. Huang CCY, Ma T, Roltsch Hellard EA, Wang X, Selvamani A, Lu J, et al. Stroke triggers nigrostriatal plasticity and increases alcohol consumption in rats. Sci Rep. 2017;7:2501.

19. Gong S, Doughty M, Harbaugh CR, Cummins A, Hatten ME, Heintz N, et al. Targeting Cre recombinase to specific neuron populations with bacterial artificial chromosome constructs. J Neurosci. 2007;27:9817-23.

20. Madisen L, Mao T, Koch H, Zhuo JM, Berenyi A, Fujisawa S, et al. A toolbox of Credependent optogenetic transgenic mice for light-induced activation and silencing. Nat Neurosci. 2012;15:793-802.

21. Cruikshank SJ, Urabe H, Nurmikko AV, Connors BW. Pathway-specific feedforward circuits between thalamus and neocortex revealed by selective optical stimulation of axons. Neuron. 2010;65:230-45.

22. Ding J, Peterson JD, Surmeier DJ. Corticostriatal and thalamostriatal synapses have distinctive properties. J Neurosci. 2008;28:6483-92.

23. Mateo $\mathrm{Y}$, Johnson KA, Covey DP, Atwood BK, Wang $\mathrm{HL}$, Zhang $\mathrm{S}$, et al. Endocannabinoid actions on cortical terminals orchestrate local modulation of dopamine release in the nucleus accumbens. Neuron. 2017;96:1112-26 e5.

24. Bamford NS, Zhang H, Schmitz Y, Wu NP, Cepeda C, Levine MS, et al. Heterosynaptic dopamine neurotransmission selects sets of corticostriatal terminals. Neuron. 2004;42:653-63.

25. Yin $\mathrm{HH}$, Lovinger DM. Frequency-specific and D2 receptor-mediated inhibition of glutamate release by retrograde endocannabinoid signaling. Proc Natl Acad Sci USA. 2006;103:8251-6.

26. Zucker RS, Regehr WG. Short-term synaptic plasticity. Annu Rev Physiol. 2002;64:355-405.

27. Oldenburg IA, Ding JB. Cholinergic modulation of synaptic integration and dendritic excitability in the striatum. Curr Opin Neurobiol. 2011;21:425-32.

28. Higley MJ, Gittis AH, Oldenburg IA, Balthasar N, Seal RP, Edwards RH, et al. Cholinergic interneurons mediate fast VGluT3-dependent glutamatergic transmission in the striatum. PLoS ONE. 2011;6:e19155.

29. Klapoetke NC, Murata Y, Kim SS, Pulver SR, Birdsey-Benson A, Cho YK, et al. Independent optical excitation of distinct neural populations. Nat Methods. 2014;11:338-46.

30. Kravitz AV, Tye LD, Kreitzer AC. Distinct roles for direct and indirect pathway striatal neurons in reinforcement. Nat Neurosci. 2012;15:816-8.

31. Cui G, Jun SB, Jin X, Pham MD, Vogel SS, Lovinger DM, et al. Concurrent activation of striatal direct and indirect pathways during action initiation. Nature 2013;494:238-42

32. Perreault ML, Hasbi A, O'Dowd BF, George SR. Heteromeric dopamine receptor signaling complexes: emerging neurobiology and disease relevance. Neuropsychopharmacology. 2014;39:156-68.

33. MacAskill AF, Cassel JM, Carter AG. Cocaine exposure reorganizes cell type- and input-specific connectivity in the nucleus accumbens. Nat Neurosci. 2014; 17:1198-207.

34. Kuei-Sen Hsu C-CH, Cheng-Hsun Yang, Gean Po-Wu. Presynaptic D2 dopaminergic receptors mediate inhibition of excitatory synaptic transmission in rat neostriatum. Brain Res. 1995;690:264-68.

35. Barak S, Carnicella S, Yowell QV, Ron D. Glial cell line-derived neurotrophic factor reverses alcohol-induced allostasis of the mesolimbic dopaminergic system: implications for alcohol reward and seeking. J Neurosci. 2011;31: 9885-94.

36. Trantham-Davidson $\mathrm{H}$, Chandler LJ. Alcohol-induced alterations in dopamine modulation of prefrontal activity. Alcohol. 2015:49:773-9.

37. Xing B, Li YC, Gao WJ. Norepinephrine versus dopamine and their interaction in modulating synaptic function in the prefrontal cortex. Brain Res. 2016;1641:217-33.

38. Li YC, Yang SS, Gao WJ. Disruption of Akt signaling decreases dopamine sensitivity in modulation of inhibitory synaptic transmission in rat prefrontal cortex. Neuropharmacology. 2016;108:403-14. 